\title{
Velocity field in a high prandtl number liquid bridge
}

\author{
Shuo Yang ${ }^{1, a}$, Ruquan Liang ${ }^{1}$, Jicheng $\mathrm{He}^{1}$ \\ ${ }^{1}$ Key Laboratory of National Education Ministry for Electromagnetic Processes of Materials, Northeastern University in Shen Yang, China
}

\begin{abstract}
A numerical model is developed to investigate the velocity fields of high prandtl number liquid bridge with surface deformation under microgravity. The Navier-Stokes equations coupled with the energy conservation equation are solved on a staggered grid. In the numerical calculations, the free surface deformation and the effects of ambient air are considered. The surface deformation of liquid bridge is captured by using level set method of mass conservation. Results of lateral and axial velocities on different radius are given. The lateral and axial velocities near the free surface are larger than that near the center of liquid bridge. The lateral velocity and axial velocities on the interface tend to be uniform with the development of thermocapillary convection.
\end{abstract}

\section{Introduction}

Due to the effect of surface tension on the interface, the marangoni convection appears when the surface tension gradient exceeds the viscous force on the free surface $\mathrm{e}^{[1-2]}$. The change of surface tension caused by the variations of temperature along the free surface generates the flow called thermocapillary convection. Today, the space technology has been developed rapidly, and the buoyancy is ignored and the effect of marangoni convection becomes important under microgravity environment. Researches on thermocapillary convection have been an important topic in space science and fluid mechanics. Especially, thermocapillary convection will play a great impact on the quality of crystal growth under microgravity. In order to better research and control thermocapillary convection, the numerical simulation model for liquid bridge of half floating zone has been established to investigate this process. So many scholars have conducted related experiments and numerical simulations using the half-floating zone model without considering the effect of ambient air ${ }^{[3]}$. Recently, some scholars conducted studies to investigate flow and temperature fields in liquid bridges involving the effects of ambient air. Although the effect of the ambient air was considered, their studies also neglected the surface deformation of liquid bridge ${ }^{[4]}$. In addition, the flow characteristic has been still in debate for high Pr number liquid bridges $(\operatorname{Pr}>15)$.

\section{Physical model}

The figure 1 shows the simplified model of floating-zone crystal growing adopted in the present paper. The diameter of upper and bottom discs is $D=5.00 \mathrm{~mm}$. The aspect ratio $(H / R)$ is 1 . The Temperature difference $\left(\Delta \mathrm{T}=T_{\mathrm{t}}-T_{\mathrm{b}}\right)$ between cold and hot disk is $25 \mathrm{~K}$. Other main physical parameters are presented in Fig. 1.

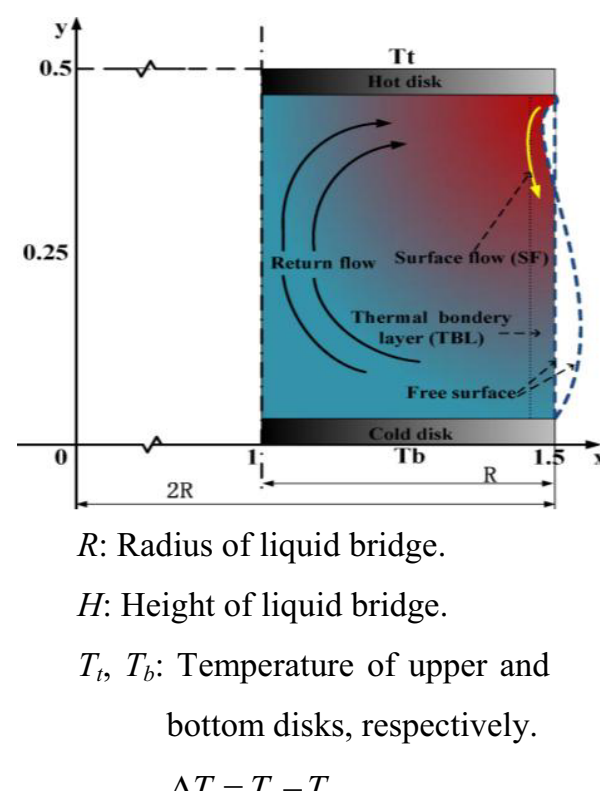

$2 R$ : Outer diameter of gas-phase region.

Figure. 1: Schematic of a half-zone liquid bridge model

\section{Controlling equations}

a S. Yang:812886674@qq.com 
The surface tension on the free interface is a linear function of temperature and decreases linearly with the temperature increasing. The basic governing equations of the half-floating zone thermocapillary convection include mass conservation, momentum conservation, and energy conservation equations. The non-dimensional governing equations are given as following:

$$
\begin{gathered}
\mathbf{u}_{t}+(\mathbf{u} \cdot \nabla) \mathbf{u}= \\
\frac{1}{\rho}\left(-\nabla p+\frac{1}{\operatorname{Re}} \nabla \cdot(2 \mu \mathbf{D})+\left(\frac{1}{\mathrm{We}}-\frac{\mathrm{Ca}}{\mathrm{We}} \Theta\right) \kappa \delta(d) \mathbf{n}\right) \\
\nabla \cdot \mathbf{u}=0 \\
\frac{\partial \Theta}{\partial t}+\nabla \cdot(\mathbf{u} \Theta)=\frac{1}{\operatorname{Re} \operatorname{Pr}} \nabla^{2} \Theta
\end{gathered}
$$

where $\mathbf{u}=(u, v)$ is the fluid velocity, $\rho=\rho(\mathbf{x}, t)$ is the fluid density, $\mu=\mu(\mathbf{x}, t)$ is the fluid viscosity, $\boldsymbol{D}$ is the viscous stress tensor, $\kappa$ is the curvature of the interface, $d$ is the normal distance to the interface, $\delta$ is the Dirac delta function, $\mathbf{n}$ is the unit normal vector at the interface, $t$ is the time, $p$ is the pressure.

The key parameters are $\operatorname{Re}=\rho_{l} U L / \mu_{l}$, Reynolds number, where $L$ is the characteristic length defined as $L=2 R$, where $R$ is the initial radius of the liquid bridge. $U$ is the characteristic velocity defined as $U=\sigma_{T} \Delta T / \mu_{l}$ in microgravity conditions, where $\sigma_{T}$ is the temperature dependency of surface tension, $\Delta T=T_{t}-T_{b}$ is the temperature difference between the top and bottom disks ; We $=U^{2} L \rho_{l} / \sigma$, Weber number; $\operatorname{Pr}=\mu_{l} / \rho_{l} a$, Prandtl number, where $a$ is the thermal diffusivity; $\mathrm{Ca}=\mu_{l} U / \sigma$, Capillary number; We denote $\sigma$ as the surface tension, $\sigma=\sigma_{c}-\sigma_{T}\left(T-T_{b}\right)$, where $\sigma_{c}$ is a reference value of surface tension, and $\Theta$ as the excess temperature, $\Theta=\left(T-T_{b}\right) / \Delta T$. Here $\rho_{l}$ and $\mu_{l}$ are the dimensional liquid density and viscosity, respectively, and the dimensionless density $\left(\rho_{l} / \rho_{l}\right)$ and viscosity $\left(\mu_{l} / \mu_{l}\right)$ inside the liquid bridge are equal to $1 . \rho_{g}$ and $\mu_{g}$ are the dimensional density and viscosity of ambient air, respectively.

\section{Results and discussion}

In the following, the analysis of velocity field in liquid bridge of high Pr number fluid under microgravity is given. The computational conditions are given as follows: the Marangoni number is $11063.99, \mathrm{M} a=\mathrm{Re} \operatorname{Pr}$, the Prandtl number is 111.7 , the Weber number is 80.6 , the Capillary number is 0.081 , the gravity level is $g=0.1 g_{0}$ $\left(g_{0}=9.81 \mathrm{~m} / \mathrm{s}^{2}\right)$, the density ratio of gas phase and liquid phases is $\rho_{l} / \rho_{g}=774$, the viscosity ratio of gas phase and liquid phases is $\mu_{l} / \mu_{g}=519.4$, the densit $\mathrm{y}$ of silicone oil is $\rho_{l}=935 \mathrm{~kg} / \mathrm{m}^{3}$, and the viscosity of silicone oil is $v$ $=9.35 \times 10^{-3} \mathrm{~Pa} \cdot \mathrm{s}$.

Figure 2 shows the distribution of lateral velocity $(u)$ at different radius. The lateral velocity $(u)$ near the free surface of liquid bridge is large, but it is small near the center of liquid bridge. It designates that the surface flow is more violent than the bulk reflux inside liquid bridge. The value of lateral velocity $(u)$ is divided into positive and negative. The positive velocity represents that the

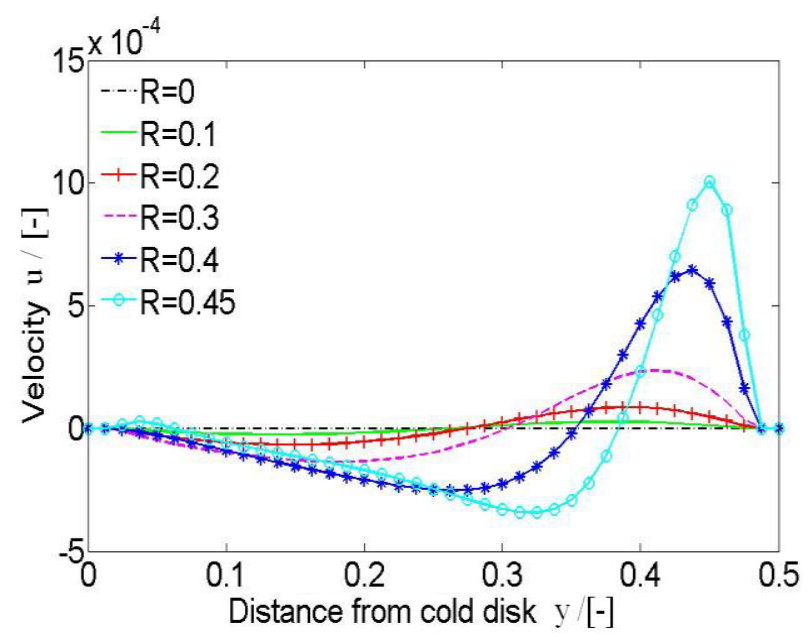

(a) $t=10 \mathrm{~s}$

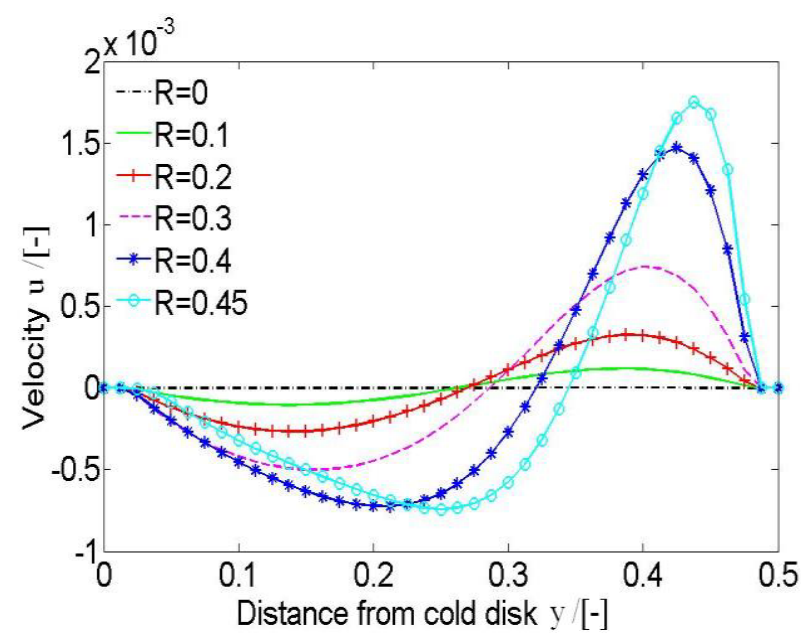

(b) $t=50 \mathrm{~s}$

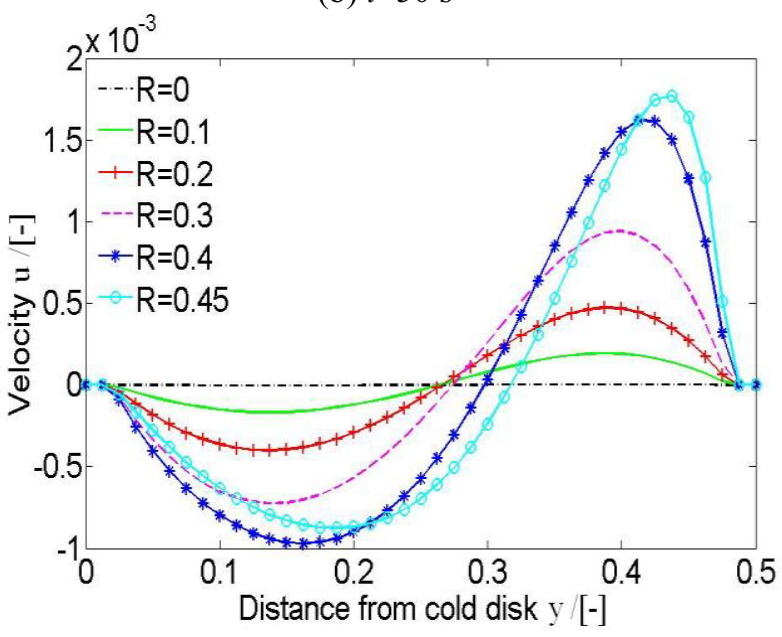

(c) $t=100 \mathrm{~s}$

Figure.2: Distribution of lateral velocity $(u)$ at different radius under $0.1 g_{0}$ gravity condition

$(\operatorname{Pr}=111.7, \quad M a=11063.99, \quad W e=80.6, \quad C a=0.081$, $\left.D=5.0 \mathrm{~mm}, H / R=1.0, g=0.1 g_{0}\right)$ 


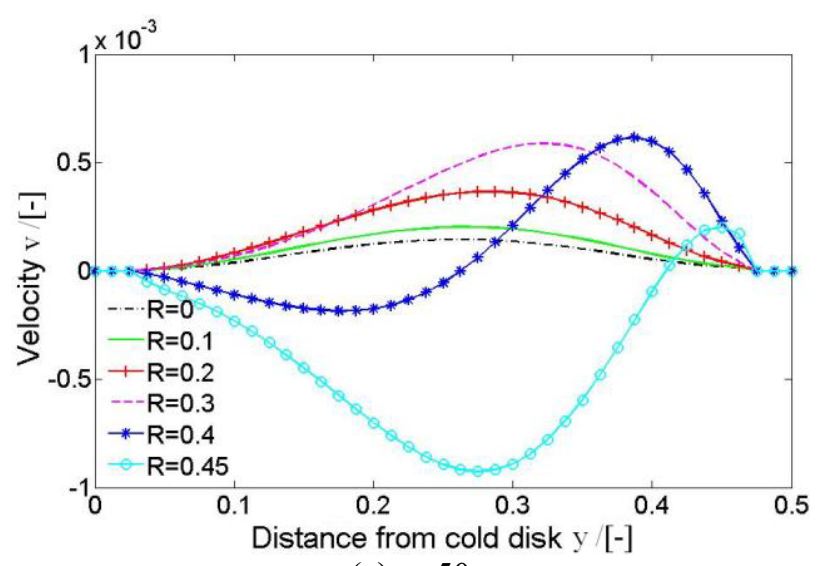

(a) $t=50$

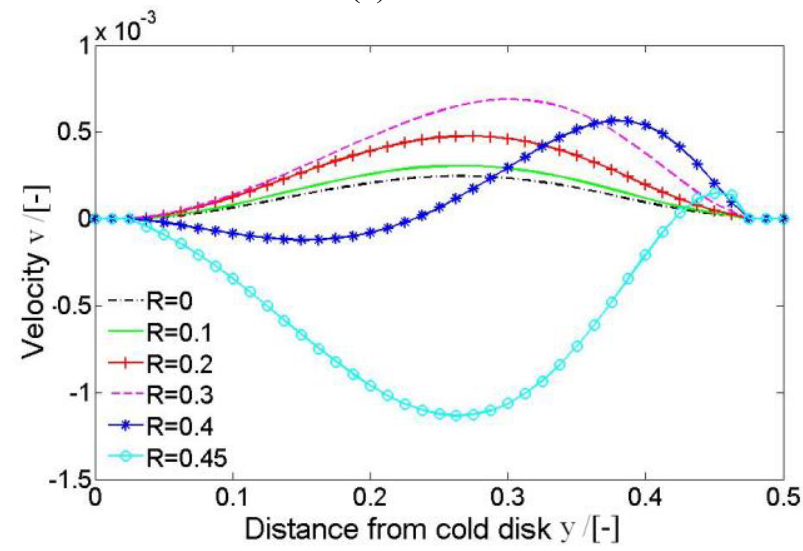

(b) $t=100$

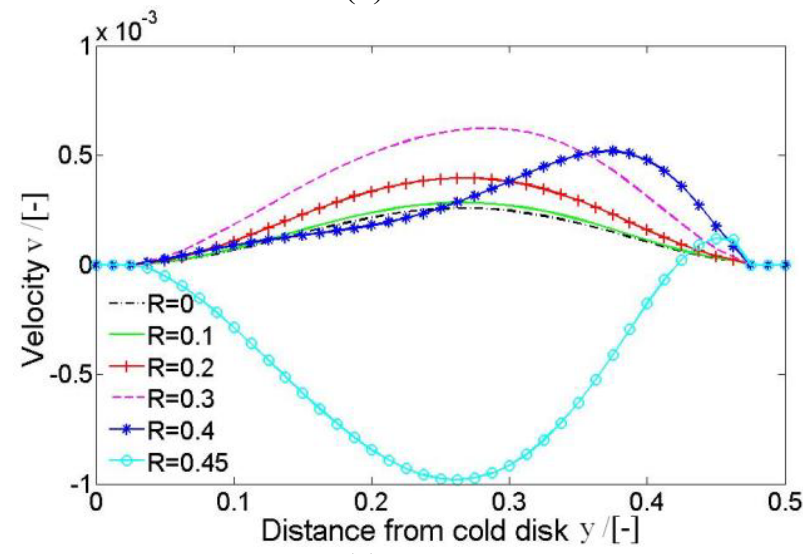

(c) $t=200$

Figure. 3: Distribution of axial velocity (v) at different radius under $0.1 g_{0}$ gravity condition

$(P r=111.7, M a=11063.99, W e=80.6, C a=0.081$, $\left.D=5.0 \mathrm{~mm}, H / R=1.0, g=0.1 g_{0}\right)$

direction of velocity $(u)$ is from the free surface to the inner of liquid bridge, and the negative velocity represents that the direction of velocity is from free surface to the inner of liquid bridge. Meanwhile, the maximum and minimum of lateral velocity $(u)$ change with the time variation. In the late stage $(t=100$ and 200), the overall level of the lateral velocity $(u)$ is improved. At the same time, the influence scope and the position of cell flow directly affect the distribution of the positive or negative velocity. When the thermocapillary convection becomes strong and moves toward the inward and the cold disk, the lateral velocity $(u)$ on the interface is uniform.
Figure 3 shows the distribution of axial velocity $(v)$ for $10 \mathrm{cst}$ silicone oil at different radius. In the early development of thermocapillary convection, the axial velocity gradient is large, and the difference between maximum and minimum is also large. With the increasing of the temperature difference, the surface flow tends to be stable and the distribution of velocity tends to be uniform.

\section{Conclusions}

1. Because the surface flow is more violent than the bulk reflux inside liquid bridge, the lateral velocity $(u)$ near the free surface of liquid bridge is larger than that near the center of liquid bridge. When the vortex center moves toward the inward and the cold disk, the lateral velocity (u) on the interface tends to be uniform with the development of thermocapillary convection.

2. In the early, the axial velocity gradient near the free surface is large. The difference between maximum and minimum is also large. When the temperature difference is increased, the surface flow tends to be stable and the distribution of the axial velocity tends to be uniform.

\section{Acknowledgements}

The present work is supported financially by the National Natural Science Foundation of China under the grants of 51376040 and 11072057.

\section{References}

1 Marangoni C. On the expansion of a drop of liquid floating in the surface of another liquid ("Sull' espansione delle gocce di un liquido gallegianti sulla superficie di altro liquido"). (Pavia, Italy: Tipographia dei fratelli Fusi), (1865)

2 Willard Gibbs J, Transactions of the Connecticut. Academy of Arts and Sciences, On the equilibrium of heterogeneous substances. 3, 343-524 (1878)

3 Cheng M., Kou S, J. Cryst. Growth, Detecting temperature oscillation in a silicon liquid bridge. 2, 132-135 (2000)

4 Bazzi H., Nguyen C. \& Galanis N.. Int. J. Therm. Sci, Numerical study of the unstable thermocapillary flow in a silicon float zone under $\mu-g$ condition. 4, 702716, (2001) 